\title{
Salidas pedagógicas con niños y niñas de 2 a 4 años en Bogotá: una mirada desde la educación inicial
}

Volumen 6 N.o 48 enero-junio de 2020

ISSN: 0122-4328

ISSN-E: 2619-6069 pp. $57-67$
Pedagogical Outings with Boys and Girls from 2 to 4 Years Old in Bogotá: a View From the Initial Education

Luz Kathy González Rodríguez*

\author{
Passeios \\ pedagógicos com \\ meninos e meninas \\ de 2 a 4 anos em \\ Bogotá: uma vista da \\ educação inicial
}

Fecha de recepción: 02-03-20

Fecha de aprobación: 06-04-20 


\section{RESUMEN}

Una inquietud compartida por las maestras de educación inicial versa sobre las dinámicas sociales en las que se desenvuelven los niños y niñas en la ciudad, las cuales suelen restringirse a entornos arquitectónicamente cerrados. En este artículo de investigación se analizan las salidas pedagógicas como alternativa frente a dicha problemática, desde la perspectiva de algunas maestras y la caracterización de propuestas no convencionales privadas de Bogotá para niños y niñas entre 2 y 4 años. La investigación se propuso desde la postura epistemológica pragmática de tipo descriptivo exploratorio. Como estrategia de investigación se asumió la etnografía rápida, a través de la cual se contó con la participación de maestras y una experta, así como con la información recogida a partir de la exploración documental de información secundaria y la visita a algunas entidades privadas que ofrecen educación no convencional a niños y niñas de 2 a 4 años en Bogotá. El marco teórico se estructura alrededor de tres categorias: pedagogía urbana, ciudad educadora y educación inicial. Como principales hallazgos se encuentra el valor preponderante que las maestras otorgan a las salidas pedagógicas como parte de sus apuestas pedagógicas con los niños y niñas. En segundo lugar, están las oportunidades y perspectivas de las salidas pedagógicas, dentro de las cuales se encuentran la posibilidad de aportar al desarrollo de los niños y niñas, y la comprensión de la ciudad como contenido, medio y fin educativo. También se encuentran reflexiones relacionadas con la educación para la diversidad y los entornos naturales como reto de ciudad para los niños y niñas.

Palabras clave: educación infantil; pedagogía urbana; salidas pedagógicas; primera infancia; ciudad educadora

\section{ABSTRACT}

A concern shared by early childhood teachers is about the social dynamics in which boys and girls develop in the city, which are usually restricted to architecturally closed environments. This research article analyzes the pedagogical outings as an alternative to this problem, from the perspective of some teachers and the characterization of private unconventional proposals in Bogotá for boys and girls between 2 and 4 years old. The research was proposed from the pragmatic epistemological posture of an exploratory descriptive type. As a research strategy, rapid ethnography was assumed, through which teachers and an expert participated, as well as the information collected from the documentary exploration of secondary information and the visit to some private institutions that offer unconventional education to boys and girls from 2 to 4 years old in Bogotá. The theoretical framework is structured around three categories: urban pedagogy, educating city and initial education. As main finding is identified the preponderant value that teachers give to educational outings as part of their pedagogical intentions with boys and girls. Second, there are the opportunities and perspectives of pedagogical outings, among which are the possibility of contributing to the development of children, and the understanding of the city as an educational content, means and purpose. There are also reflections related to education for diversity and natural environments as a city challenge for boys and girls.

Keywords: early childhood education; urban pedagogy; pedagogical outings; early childhood; educating city

\section{RESUMO}

Uma preocupação compartilhada por professores de educação infantil é sobre a dinâmica social em que meninos e meninas se desenvolvem na cidade, geralmente restrita a ambientes arquitetonicamente fechados. Este artigo de pesquisa analisa os passeios pedagógicos como alternativa a este problema, a partir da perspectiva de algumas professoras e da caracterização de propostas não convencionais privadas em Bogotá para meninos e meninas entre 2 e 4 anos de idade. A pesquisa foi proposta a partir da postura epistemológica pragmática de tipo exploratório descritivo. Como estratégia de investigação, assumiu-se a etnografia rápida, através da qual participaram docentes e um perito, bem como as informações recolhidas a partir da exploração documental de informação secundária e da visita a algumas entidades privadas que oferecem formação não convencional para meninos e meninas de 2 a 4 anos em Bogotá. 0 referencial teórico está estruturado em torno de três categorias: pedagogia urbana, cidade educadora e educação inicial. Como principal constatação está o valor preponderante que os professores atribuem às saídas educativas como parte das suas apostas pedagógicas com meninos e meninas. Em segundo lugar, estão as oportunidades e perspectivas dos passeios pedagógicos, entre as quais a possibilidade de contribuir para o desenvolvimento das crianças e a compreensão da cidade como conteúdo, meio e finalidade educacional. Há também reflexões relacionadas à educação para a diversidade e os ambientes naturais como um desafio da cidade para meninos e meninas.

Palavras-chave: educação infantil; pedagogia urbana; passeios pedagógicos; primeira infância; cidade educadora 


\section{Introducción}

Las salidas pedagógicas son una de las posibilidades que tienen los jardines infantiles y las instituciones educativas para proporcionar una vivencia pedagógica diferente a la que ofrecen cotidianamente, favoreciendo que los niños y niñas tengan una experiencia de recorrido y conocimiento de la ciudad enriquecida gracias al quehacer pedagógico de la maestra', quien le otorga unas intencionalidades, organización, metodología y contenidos sobre los cuales focaliza y estructura la visita a dichas propuestas en la ciudad.

Este artículo recoge los análisis de una investigación ${ }^{2}$ cuyo objeto de estudio son las salidas pedagógicas que realizan los jardines infantiles y las instituciones educativas que atienden niños y niñas entre los 2 y 4 años, ${ }^{3}$ en relación con la oferta educativa no convencional ${ }^{4}$ de carácter privado ${ }^{5}$ de la ciudad de Bogotá y que, dentro de su diseño y propuesta de atención, contemplan estas edades.

En primer lugar, se presenta el contexto que origina la reflexión sobre la importancia de las salidas pedagógicas, enseguida la metodología empleada, y luego los principales resultados y análisis de la caracterización de dicha oferta educativa no convencional, a partir de la identificación de sus intencionalidades, contenidos y metodologías educativas, así como de las características, usos y aportes que ofrecen las salidas pedagógicas. Por último, se comparten las principales conclusiones del estudio.

1 A lo largo del documento se hablará de maestras atendiendo a que la mayor parte de las personas a cargo de la educación de los niños y niñas en primera infancia en las instituciones educativas de Bogotá pertenece al sexo femenino. Esta decisión no excluye que se encuentren hombres incursionando en este campo y en esa medida el contenido de este artículo resulte de su interés. Se sugiere asumir que el término acoge a los dos sexos.

2 Enmarcada en los desarrollos del Grupo de Pedagogia Urbana y Ambiental, de la Maestría en Educación de la Universidad Pedagógica Nacional.

3 Se definió el periodo de 2 a 4 años al no haber sido ampliamente explorado por investigaciones previas afines a este estudio, además corresponde a un momento de vida de los niños y las niñas, en el que aumentan sus posibilidades de desplazamiento y actividad autónoma, lo cual favorece que las maestras tengan mayor interés en involucrarlos en salidas pedagógicas.

4 Lo no convencional está asociado a la oferta educativa no formal, la cual tiene unas intencionalidades y organización diferentes a las tradicionales (asociadas al ámbito escolar), sus propuestas no dan lugar a la obtención de certificados, y son flexibles en sus contenidos y metodologías.

5 Solo fueron objeto de análisis las propuestas de carácter privado, porque la oferta pública de Bogotá se ha venido incrementando y su estudio en profundidad requería de un tiempo y una dedicación mayores.

\section{Contexto de la problemática}

En la actualidad, la interacción de las personas en el espacio público de la ciudad no se da con la misma naturalidad y tranquilidad que ocurría hace unas décadas, cuando muchos eventos de tipo social, cultural y económico se convertían en pretexto para el encuentro y la socialización de las personas. Dicha experiencia ha venido cambiando, como ha sido señalado por Páramo y Cuervo (2009), ${ }^{6}$ quienes afirman que las prácticas de vida en Bogotá han transitado por una serie de variaciones fruto de las diversas construcciones de significados que han tenido lugar en la ciudad.

Esta situación tiene mayor impacto en los niños y niñas, quienes por las dinámicas laborales de los adultos permanecen largas jornadas del día en sus casas o en instituciones de educación inicial, ${ }^{7}$ al ser estos los primeros y principales entornos en los que transitan y donde tienen posibilidades de encuentro e interacción con otros. Esto hace que las oportunidades de explorar el espacio público y los lugares privados de encuentro social con vocación colectiva (Páramo y Cuervo, 2009) estén sujetas a la voluntad e iniciativa de los adultos, quienes por falta de tiempo, desconocimiento o coaccionados por el miedo procuran evitarlas, limitando así las posibilidades de socialización, descubrimiento, interacción y aprendizaje que pueden tener lugar en tales espacios.

Este resulta ser un problema de especial trascendencia dada la importancia que tiene para los seres humanos la primera infancia, entendida en el Artículo 29 del Código de Infancia y Adolescencia (2006) como la "etapa del ciclo vital en la que se establecen las bases para el desarrollo cognitivo, emocional y social del ser humano, la cual comprende la franja poblacional que va de los cero (0) a los seis (6) años de edad", de manera que lo que ocurra en este periodo de vida tiene especial valor tanto por la carga experiencial que supone para el niño y la niña, como por el impacto para su vida futura.

\footnotetext{
6 En su estudio del año 2006 sobre la historia social situada en el espacio público desde su fundación hasta el siglo xx.

7 Dentro de las cuales se encuentran jardines infantiles, hogares comunitarios del ICBF, centros de desarrollo infantil y algunos colegios públicos y privados.
} 
Por ello, contar con iniciativas en las que los niños y niñas de primera infancia accedan a experiencias educativas fuera de los entornos habituales donde transcurren sus vidas (hogar e institución educativa) plantea un reto importante para la ciudad y, de manera especial, para los adultos, quienes tienen la responsabilidad de garantizar las condiciones necesarias para que su participación salga de esos espacios y se traslade al espacio público, de manera que se superen las barreras que impone el "miedo a la ciudad"8 (Páramo, 2007) en las personas y de manera especial durante esta etapa de vida.

De igual manera, las investigaciones realizadas desde finales del siglo xx demuestran que cada vez toman más fuerza las iniciativas educativas que se desarrollan o utilizan recursos educativos ubicados fuera del aula, asumiendo que la ciudad puede convertirse en un entorno susceptible de ser aprovechado como recurso, fin y medio educativo, como se propone en los estudios de la pedagogía urbana (Colom, 1996). Este planteamiento se ubica en una perspectiva optimista de la ciudad (Páramo, 2004) que resulta afín a las investigaciones realizadas por Carpenter y McLuhan (1974), quienes argumentan la pertinencia de realizar experiencias en el espacio exterior al aula, buscando recuperar y darle relevancia a las prácticas y recursos educativos que están allí.

\section{Metodología}

La investigación se propuso desde la postura epistemológica pragmática y fue de tipo descriptivo exploratorio. Como estrategia de investigación se seleccionó la etnografía rápida, mediante la cual se contó con la participación de maestras a través de un cuestionario virtual y de una experta en el tema a través de una entrevista, así como con información recogida a partir de la exploración documental de información secundaria y una observación de las entidades con oferta educativa no convencional de carácter privado dispuestas para los niños y las niñas de 2 a 4 años, en el área metropolitana de la ciudad de Bogotá.

8 "Durante mucho tiempo se hizo predominante en la investigación urbana identificar los aspectos negativos de vivir en las grandes ciudades. Los trabajos de la sociología y la psicología durante buena parte del xx sugieren que muchas de las condiciones de la vida urbana como la alta densidad poblacional, el ruido, la polución, la necesidad de hacer uso de transporte masivo tienen la capacidad de generar estrés". (Páramo, 2007, s. p.)

\section{Resultados y análisis}

\section{Salidas pedagógicas, experiencia vital en la educación inicial en Bogotá}

Las salidas pedagógicas son un tipo de experiencia educativa que suele integrarse a los procesos curriculares que realizan las maestras de todos los niveles educativos. Es muy valioso encontrar que, de las 36 maestras participantes en la investigación, solo una manifestó que en su institución educativa no adelantan este tipo de vivencias, debido al temor a la inseguridad, mientras que todas las demás implementan entre 1 y 10 salidas al año, siendo 2 al año el número más alto en las respuestas, correspondiente a la mitad de las maestras participantes.

En coherencia con los planteamientos de Páramo (2004), este hallazgo derivado de las encuestas se convierte en una evidencia de que las maestras participantes han ido alimentando una perspectiva positiva de la ciudad, pues reconocen las experiencias positivas que ofrece el ambiente urbano, y encuentran en la ciudad diferentes beneficios para los niños y niñas, es decir que, su comprensión sobre esta supera la concepción tradicional de la urbe como un espacio físico, y le otorga valores educativos, afectivos, culturales y sociales, venciendo los temores y prevenciones que sobre ella circulan.

En este mismo sentido, se identifica que desde la perspectiva de las maestras las salidas pedagógicas promueven el desarrollo infantil, favorecen el aprendizaje de diferentes tipos de temáticas (en oportunidades relacionadas con las propuestas educativas que las maestras llevan a cabo en el aula) y suscitan la interacción de niños y niñas con sus pares y adultos nuevos o distintos a los que les proporcionan cotidianamente atención y cuidado.

De manera complementaria a este reconocimiento que hacen las maestras sobre la oferta pública que tiene Bogotá, y pese a la existencia de retos importantes relacionados con que esta ciudad siga avanzando para que sea más incluyente, ambientalmente sostenible y democrática, la Carta de las Ciudades Educadoras (Asociación Internacional de Ciudades Educadoras, 2009) reconoce que la capital del país ofrece posibilidades educativas importantes. Como parte de dichas 
oportunidades, se destaca que tiene un carácter diverso y multicultural, es favorecedora de la identidad local, promueve la convivencia, la participación ciudadana y la exploración del espacio público, y cuenta con orientaciones hacia la formación integral de los ciudadanos (Moncada, citado por Muñoz, 2015, p. 7).

De otro lado, la valoración positiva de las salidas pedagógicas por parte de las maestras y la experta consultada ${ }^{9}$ se interpreta como un avance importante que está dando la ciudad en cuanto a la educación inicial, y en ello cobran gran relevancia las orientaciones pedagógicas con que cuenta en la actualidad. Tales orientaciones provienen de la Secretaría de Educación Distrital, SED (2015), la Secretaría Distrital de Integración Social, SDIS (2009) y el Ministerio de Educación Nacional (MEN), quienes señalan la importancia de valerse de los recursos del medio para el desarrollo y fortalecimiento de los procesos educativos con los niños y las niñas:

Esta experiencia de actuar y de relacionarse en el tiempo y en el espacio con las personas, objetos, situaciones, sucesos y contextos, propicia un proceso de construcción de sentido de lo que es y pasa en el mundo, y de lo que implica habitar en él. Esta construcción de sentido, en la que intervienen las capacidades con las que se nace es un proceso reciproco: las niñas y los niños significan y dan sentido al mundo en el que viven $y$, a su vez, ellas y ellos se van conformando como sujetos del mundo a partir de lo que reciben de él. (MEN, 2014, p. 13)

Esto también explica el hallazgo respecto a la oferta, pues a pesar de no ser muy amplia en términos cuantitativos (solo se encontraron 12 entidades ${ }^{10}$ ), sí permite reconocer la construcción de sentido que allí se ha dado en torno a las experiencias que requieren los niños y niñas más pequeños, lo cual se hace evidente a través de sus propuestas. En ellas aparece con claridad la intencionalidad de trabajar con niños y niñas de 2 a 4 años, se proponen alternativas educativas adaptadas a sus características, así como materiales y, en algunos casos, incluso personal especializado para el acompañamiento.

9 Profesional especializada de la Secretaría de Educación Distrital.

10 Para el año 2016, 3 correspondian a museos (25\%), otras 3 a parques temáticos $(25 \%), 2$ a teatros de títeres y otras funciones artísticas (16\%) y las restantes 4 a oferta de diversa indole (33\%).
No obstante, también es preciso tener presente como hallazgo que en las instituciones educativas oficiales es mucho menos frecuente la realización de este tipo de salidas, lo cual probablemente está relacionado con el poco tiempo que tiene de ofertarse la educación inicial en los colegios distritales, ${ }^{11}$ así como por los factores asociados a seguridad, costos y oferta.

\section{Recorridos por la ciudad, oportunidades y perspectivas}

Una vez identificada la realización de salidas pedagógicas como una iniciativa que tiene un alto nivel de prioridad en las maestras participantes, resulta relevante analizar los elementos que ellas consideran constitutivos y destacables sobre la realización de este tipo de experiencias:

\section{Desarrollo infantil, un propósito compartido}

Promover el desarrollo infantil es la primera razón que las maestras expresaron para realizar salidas pedagógicas, revelando, además, que empieza a tejerse un lazo entre tres ámbitos: jardines infantiles o instituciones educativas, oferta educativa no convencional e institucionalidad, ${ }_{1}^{12}$ los cuales comparten sentidos alrededor de dicho objetivo.

De igual manera, se encuentra afinidad entre el discurso de la oferta y la demanda, el cual se posiciona y evidencia a partir de lo que ha venido llamándose "dimensiones", las cuales son una forma de organización para que desde la comprensión del niño y la niña como sujetos integrales, se observen y acompañen sus procesos de desarrollo, a través de sus diferentes manifestaciones: corporales, comunicativas, cognitivas, sociales, artísticas, etc. Como expresan Woodhead y Oates (2007): "El desarrollo posee varias dimensiones interrelacionadas y el aprendizaje se da en cada una de ellas. Por ende, hay que considerar el desarrollo y el aprendizaje holisticamente y las intervenciones deben prestar al niño una atención integral" (p. 2).

11 La oferta de educación inicial en los colegios oficiales del Distrito no cuenta con más de dos años de trayectoria, no se está ofreciendo en todas las instituciones y las experiencias institucionales que están en curso, se encuentran en proceso de construcción de sus propuestas pedagógicas para estas edades.

12 Entendida como las instituciones encargadas de acompañar y supervisar los procesos educativos en la ciudad y el país: SED, SDIS, MEN. 
En consecuencia, encontrar que el desarrollo empieza a hacer parte de un propósito común entre la escuela y la ciudad es un aliciente para que continúen realizándose este tipo de experiencias, de forma que la oferta educativa no convencional se cualifique, vincule a otros sectores $y$, de la mano de las maestras, pueda aportar de modo progresivo a las construcciones teóricas sobre la educación inicial y la pedagogía urbana.

Como elemento final respecto a este componente, es fundamental señalar que la expresión referida a la garantía del derecho a la educación inicial no se restringe solo a la posibilidad de que los niños y niñas accedan a este servicio, sino que este se preste contemplando de forma intencionada toda una serie de elementos que lo enriquecen y responden a este momento socio-histórico particular, en el que el desarrollo humano cobra especial relevancia, y sobre el que las maestras hacen un claro llamado en sus respuestas: "lo más importante de estas salidas es permitir a los niños ser los protagonistas, que puedan explorar y recibir talleres que los estimule y ayude a desarrollarse adecuadamente en cada una de sus etapas" (informante maestra 3, mujer, 2014).

\section{La ciudad como contenido, medio y fin educativo}

Una vez clara la importancia que tiene la interrelación entre los discursos que circulan en torno al aporte principal de las salidas pedagógicas, se presenta otro elemento constitutivo de la investigación, el cual, desde la pedagogía urbana tiene que ver con lo que Colom plantea como comprender la ciudad como escenario de formación (Páramo, 2009).

Las maestras y las propuestas de oferta educativa no convencional de carácter privado para niños y niñas entre los 2 y 4 años identifican una serie de contenidos que vinculan lo que ocurre en el entorno educativo y lo que puede ofrecer la ciudad, tal como se propone desde la pedagogía urbana. Así, tanto las maestras como la oferta destacan que a través de las salidas puede hacerse una incorporación, o abordaje de contenidos o temáticas que enriquezcan o complementen los espacios educativos, asociados principalmente a las artes, la ciencia y la literatura. De esta manera, se asume que, a través del contacto directo y experiencial con la ciudad, esta última se convierte en un recurso informativo, o "un medio didáctico o instrumental que puede facilitar el aprendizaje de cuestiones integradas a los planes de estudios escolares" (Colom, citado por Páramo, 2009, p. 21).

De ahí que las entidades reguladoras de la prestación del servicio sugieran con insistencia la importancia de vincular la salida o "expedición pedagógica" a las temáticas o proyectos pedagógicos que se realizan con los niños y niñas, pues se asume que la oferta educativa no convencional tiene la posibilidad de proporcionar elementos nuevos, complementarios y de refuerzo de los contenidos curriculares propios de la educación inicial que favorecen el aprendizaje. Esto fue señalado por las maestras a través de expresiones como: "estos lugares les permite a los niños(as), explorar, indagar, cuestionarse entre otras, lo que les admite un aprendizaje significativo" (informante maestra 3, mujer, 2014).

Conforme a lo anterior, se identifica que son diversas las potencialidades educativas de la oferta estudiada, en la medida en que en esta se visibilizan con claridad elementos o posibilidades que surgen en el marco de las ciudades educadoras, las cuales se acogen desde la pedagogía urbana y fueron señaladas por Páramo (2009), citando a Colom y Trilla de la siguiente manera:

1. "La ciudad como entorno o contenedor de recursos educativos o 'aprender en la ciudad', propone que la urbe cuenta con elementos importantes para ser considerada un objeto de aprendizaje" (Páramo, 2009, p. 21). De esta manera, que en Bogotá existan entidades con una serie de recursos, equipamientos, medios y eventos de carácter educativo (Trilla, 2005) orientados a la realización de salidas pedagógicas con niños y niñas, que estos se acompañen de personas cualificadas y que allí tengan lugar acontecimientos y encuentros educativos planeados y no planeados educativamente (Muñoz, 2015), como los encontrados en esta investigación, da cuenta de la existencia de una parte importante de las condiciones necesarias para que Bogotá se convierta en una ciudad educadora. 
2. La ciudad como agente educativo o "aprender de la ciudad" comprende el medio urbano como un agente educativo informal. Este componente se identificó en los resultados en tanto a través de las salidas los niños y niñas se aproximan a un entorno diferente a sus referentes cercanos, lo cual da lugar al aprendizaje de comportamientos, normas y capacidades que solo alli se requieren, permitiendo la integración de elementos culturales, cívicos, reglas, valores y tradiciones a manera de un "currículo oculto" de la ciudad (Trilla, 2005).

3. La ciudad como contenido u objetivo educativo o "aprender la ciudad". Este elemento hace alusión a la posibilidad que tienen las personas de conocer, apropiarse e identificarse con la ciudad, y es señalado en los resultados en tanto a través de las salidas pedagógicas los niños y niñas entre 2 y 4 años abren su espectro respecto a sus entornos habituales de vida, y pueden empezar a identificar íconos de ciudad y elementos significativos de esta, que les ofrecen aportes para su construcción de identidad y socialización: "Son espacios que ofrecen la posibilidad de interactuar de formas diferentes con otros, con el entorno, con las culturas" (informante maestra 10, mujer, 2014).

Es así como la realización de una salida pedagógica favorece en los niños y niñas procesos sociales, de convivencia e identidad, al empezar a sentirse parte de una ciudad, a cuidarla, respetarla, pero también a comprender las diferentes maneras y reglas para recorrerla (hacer uso del transporte, conocer y respetar las normas de tránsito, permanecer con el grupo, cuidar sus objetos personales, recoger la basura), las cuales dificilmente se aprenderian fuera de este contexto, dado que están mediadas por la vivencia y adquieren sentido solo en el lugar donde tienen aplicabilidad.

Todo lo anterior da lugar a pensar que las salidas pedagógicas con niños y niñas de primera infancia superan la realización de una visita o paseo recreativo, pues tienen un carácter de exploración, de intercambio con otros y de construcción de conocimiento que no se agota en sus contenidos. Para Trilla, la ciudad es un contenedor de una educación múltiple y diversa, positiva y negativa, que se extiende, fluye y se disemina por la mayoría de sus espacios, con innumerables recursos y estímulos de formación, que conforman el entramado educativo que es la ciudad, en donde se entremezclan la educación formal, no formal e informal (Páramo, 2009).

\section{El encuentro con otros, un motivo importante para salir}

Otro aspecto importante para destacar de las salidas pedagógicas tiene que ver con el encuentro y vivencia en colectivo. Esto resulta congruente con los principios expuestos por Páramo (2009) respecto a la pedagogía urbana, en la medida en que supone entender que los espacios se nutren no solo por los recursos arquitectónicos que el lugar o entidad posea, sino sobre todo por las experiencias y contacto que esta propicia.

Se resalta así la trascendencia que se le otorga al componente socializador de las salidas pedagógicas, pues los participantes señalaron que este tipo de actividad involucra habilidades importantes relacionadas con la oportunidad de interactuar, es decir de encontrarse, dialogar, jugar y aprender, entre otras, con otras personas (guías, pares, público), y que dichos encuentros promueven la convivencia, la resolución de problemas y el trabajo en equipo.

En el campo social, para un niño de hoy en día, es casi imposible vivir el riesgo y la emoción de encontrarse con niños desconocidos con los que experimentar las delicadas estrategias del acercamiento, del conocimiento $y_{\text {, }}$ a veces, del rechazo. Sus compañeros de juego son casi exclusivamente sus compañeros de clase, sus compañeros de las clases extraescolares o los hijos de los amigos de los padres: amistades controladas y controlables por parte de los adultos. (Tonucci, 2009, p. 153)

Así, a pesar de que el "temor en la ciudad" y los "discursos del terror" (Páramo y Roa, 2015) han sido masificados por los medios de comunicación y tienen un impacto importante en las familias y maestras, las participantes en este estudio demuestran que esta condición puede ser contrarrestada y superada. ${ }^{13}$

1324 de las 36 maestras consideran que la oferta educativa no convencional de carácter privado que han visitado en la ciudad de Bogotá cuenta con buenas instalaciones y materiales adecuados y seguros para los niños. 


\section{Explorar metodologías que propicien el} aprendizaje y desarrollo infantil

Existe un marco común desde el cual se asume que las experiencias educativas que se realizan fuera del aula suelen tener componentes educativos mediados por la lúdica, el juego, el arte, la exploración, la diversión y el entretenimiento. Esto se verifica en la investigación en tanto se percibe que una salida pedagógica se convierte en sí misma en una iniciativa a través de la cual la maestra pretende lograr sus propósitos formativos con los niños y las niñas, incorporando variados elementos metodológicos: talleres, observación, interacción con módulos temáticos, recorridos guiados, exploración libre, resolución de problemas en equipo, entre otros.

Sin embargo, paralelamente las maestras participantes hacen un llamado de atención sobre el papel de los guias o responsables de la visita por parte de la oferta educativa no convencional de carácter privado, señalando la necesidad de que enriquezcan el lenguaje utilizado y las metodologías que emplean para hacerlos más cercanos a las caracteristicas del desarrollo de los niños y niñas entre los 2 y 4 años.

Esto se verificó a través de la observación, pues si bien algunas entidades empiezan a explicitar su preocupación y avances al respecto, la práctica demuestra que el reto es grande, el acercamiento a los niños y niñas sigue siendo tímido y un tanto arraigado en los estereotipos sobre lo que se piensa que ellos hacen y les gusta. En esa medida, es claro que, como propone Tonucci (2004), para el trabajo pedagógico con niños y niñas en primera infancia se requiere de aliados que apoyen su exploración de la ciudad, de manera que resulta indispensable la selección y formación del personal en torno a las características de estas edades, para que las metodologías y recursos comunicativos resulten acertados y pertinentes.

De otra parte, se encuentra el papel del maestro, sobre quien se destaca su liderazgo en la salida, sugiriendo que, si bien la oferta tiene que cualificar la atención que ofrece a través de las personas guía o acompañantes, es el maestro quien debería asumir el liderazgo durante todo el proceso, de manera que la selección del lugar, la visita y posterior análisis de la experiencia esté a su cargo, al ser la persona con conocimiento y experticia respecto a los procesos educativos de su grupo de niños y niñas, así como las intencionalidades que movilizan la salida pedagógica. Como proponen Kaufmann y Serulnicoff (2000):

si bien algunos lugares ofrecen visitas guiadas, suelen ser pocos los que cuentan con visitas apropiadas para la edad de los chicos del jardín. Por ello es aconsejable que el docente releve todas las informaciones pertinentes para luego organizar y coordinar él mismo la salida. (p. 51)

El que las maestras decidan asumir este papel, les permitirá, en compañía de niños, niñas, familias y profesionales ocupados en la tarea de educar o atender a la primera infancia, abordar y solucionar colectivamente las preguntas propuestas por Colom (citado por Páramo, 2009) sobre: ¿cuál es el aporte de la educación para solucionar los problemas presentes en la ciudad? y ¿qué ofrece la ciudad para aportar a las dificultades que afronta la educación?, entendiendo que ellas tienen mucho por aportar a la educación inicial y por qué no a la pedagogía urbana, en tanto tienen una serie de saberes construidos a través del ejercicio profesional, sus estudios realizados y la experiencia individual allí involucrada (Tardif, 2004).

\section{Educación para la diversidad}

De otro lado, un elemento que emerge como reto para la oferta educativa estudiada tiene que ver con el avance hacia la construcción de propuestas educativas incluyentes, es decir, que sus instalaciones, recursos, metodologías, contenidos e intervenciones estén preparados para atender la diversidad de niños y niñas que integran las instituciones educativas de la ciudad y se eviten las barreras que impiden su participación.

Dichas barreras u obstáculos pueden identificarse en la ausencia de rampas, espacios para el desplazamiento de sillas de ruedas, falta de señalización, utilización de un lenguaje con términos especializados que no resultan comprensibles para los niños y niñas más pequeños, disposición de materiales o información de difícil acceso, manejo o comprensión para los niños y niñas, o sencillamente en el ingreso condicionado por costos que hagan inviable el acceso de cualquier tipo de población de la ciudad. 
En ese orden de ideas, el reto que podría afirmarse trasciende a todos los escenarios educativos de la ciudad -de carácter formal, no formal e informal-, en el marco de las ciudades educadoras (Trilla, 2005) sigue siendo pensar estrategias que faciliten la participación activa de todos los niños y niñas, independientemente de sus posibilidades económicas, sociales y culturales, si tienen 2 o 7 años, o si cuentan con alguna condición de discapacidad o talentos que los hagan excepcionales. Para lograrlo, hace falta tanto voluntad como conocimiento sobre el tema, pues es un asunto que implica hacer transformaciones de fondo y forma, sobre todo porque significa una transformación profunda en términos de cultura.

\section{Entornos naturales, un reto de ciudad para los niños y las niñas}

Este aspecto se ubica como último componente del análisis, pero reporta un valor muy importante pues recoge un sentir que las maestras, la institucionalidad y la academia señalan como trascendental: la necesidad de contar con más espacios o entornos naturales dispuestos para la exploración por parte de los niños y las niñas.

Así, se encontró que las maestras privilegian las granjas y parques como dos de las primeras opciones a la hora de seleccionar los lugares que visitarán, destacando que estos posibilitan la "interacción con animales, espacio al aire libre, contacto con la naturaleza" (informante maestra 8, mujer, 2014). Tal decisión se explica por las posibilidades que este tipo de entornos le ofrecen a niños y niñas de explorar ambientes naturales y abiertos en los que pueden estar en contacto con animales y plantas. Interés que se alimenta por la inquietud que genera el que pasen buena parte de sus vidas en espacios de concreto, cerrados y con limitaciones importantes de acceso a elementos naturales.

Al respecto, la investigación realizada por Páramo y Mejía (2004) denominada "Los parques urbanos como oportunidades para la interacción de los niños con los animales", señalan dos ventajas derivadas de los encuentros en los que hay presencia de elementos naturales, las cuales pueden asumirse como un respaldo importante de lo anterior: por un lado, ofrecen soporte emocional y seguridad, ayudan a mejorar la autoestima, la recuperación fisica y emocional y en esa medida ayudan a fomentar percepciones gratas de la ciudad y, por el otro, permiten la interacción con elementos naturales y animales (palomas, patos, ardillas, pájaros y perros) por lo cual es necesario "formular iniciativas educativas como el aumento en la disponibilidad de elementos naturales en las ciudades" (p. 28).

En este sentido, se encuentra que si bien 5 de las 12 entidades de oferta educativa no convencional de carácter privado para los niños y niñas entre 2 y 4 años cuentan con algún tipo de componente natural: granja, sala especializada o museo, así como con contenidos que abordan temáticas asociadas al conocimiento, cuidado y valoración de los recursos naturales y ambientales, estos no siempre se dan en espacios naturales y abiertos en los que los niños y niñas puedan interactuar con espontaneidad, y suelen ser restringidos y condicionados al factor económico (por ejemplo para acceder a los talleres en los que interactúan con animales).

\section{Conclusiones}

Una primera conclusión derivada del estudio es el reconocimiento que hacen las maestras sobre las salidas pedagógicas, las cuales hacen parte de sus procesos educativos con los niños y niñas, accediendo a la oferta pública y privada, y venciendo los temores que aquejan en la actualidad la permanencia y vivencias en las grandes ciudades. Sin embargo, las condiciones de seguridad siguen siendo un aspecto sobre el cual la oferta y la administración de la ciudad deben trabajar, para ofrecer mayor tranquilidad a familias y maestras.

En cuanto a las caracteristicas de la oferta educativa, se concluye que dichas entidades logran formular propuestas que coinciden en bastantes aspectos con las expectativas de las maestras. Se destaca la posibilidad de vincular sus intencionalidades y acciones con las de las maestras, dándole especial relevancia a la potenciación del desarrollo de los niños y niñas, a la cual se suman las oportunidades de aprendizaje, encuentro, socialización y trabajo colaborativo. En ese sentido, maestras e instituciones logran que a través de las salidas los niños y niñas se 
diviertan, aprendan, se aproximen a elementos naturales, disfruten de la cultura y el arte y vivencien los pilares de la educación inicial: juego, arte, literatura y exploración del medio.

También puede concluirse que el papel de la maestra es definitivo en las salidas pedagógicas, pues estas experiencias no se definen únicamente por el momento de visita en las entidades de oferta, sino que están constituidas por un proceso que incluye el antes, durante y después. En consecuencia, son ellas quienes tienen el conocimiento y experiencia requeridos (tanto de los niños y niñas como de sus propuestas pedagógicas) para tomar la decisión del lugar más conveniente para realizar la salida, hacer un acompañamiento protagónico en la visita y dar cuenta de los alcances de esta.

En cuanto a la metodología empleada, cuando se realizan salidas pedagógicas se identifica que las entidades ofrecen experiencias que promueven el aprendizaje significativo de los niños y niñas más pequeños, acudiendo a actividades lúdicas, recreativas y vivenciales de trabajo individual y grupal, y disponiendo espacios agradables y estéticamente bellos. Sin embargo, también es concluyente que sobre este último elemento las maestras y la observación realizada señalan que hay un camino por recorrer respecto a la cualificación de las personas que atienden o guian las visitas, puesto que la interacción no siempre corresponde de forma adecuada a las características del desarrollo de los niños y niñas de primera infancia, sobre todo en términos de la comunicación empleada. En este orden, otro de los grandes retos de la oferta educativa no convencional tiene que ver con contar con personal, espacios y recursos que favorezcan la participación de todos y todas, es decir, que sean espacios incluyentes reconocedores de la diversidad de infancias en la ciudad.

\section{Referencias}

Asociación Internacional de Ciudades Educadoras. (2009). Carta de las Ciudades Educadoras. http://www.edcities.org/wp-content/uploads/2013/10/CARTA-CIUDADES-EDUCADORAS_3idiomas.pdf

Carpenter, E. y McLuhan, M. (1974). El aula sin muros. Investigaciones sobre técnicas de comunicación. LAA.

Colom, A. (1996). La pedagogía urbana, marco conceptual de ciudad educadora. Aportes (45).

Congreso de la República. (8 de noviembre de 2006). Ley 1098 de 2006. Código de Infancia y Adolescencia. Diario Oficial n. 46446.

Kaufmann, V. y Serulnicoff, A. (2000). Conocer el ambiente: una propuesta para las ciencias sociales y naturales en el nivel inicial. En A. Malajovich (comp.), Recorridos didácticos en la educación inicial (pp. 25-62). Paidós.

Ministerio de Educación Nacional. (2014). Serie de orientaciones pedagógicas para la educación inicial en el marco de la atención integral. Documento 24. La exploración del medio en la educación inicial. Panamericana.

Muñoz, A. (2015). Ciudades Educadoras. Espacios permanentes de aprendizaje.

Páramo, P. (2004). Algunos conceptos para una perspectiva optimista de vivir la ciudad. Territorios, 11, 91-109.

Páramo, P. (2007). La ciudad: una trama de lugares. Psicología para América Latina, (10). http:// pepsic.bvsalud.org/scielo.php?script=sci_arttext\&tpi$d=$ S1870-350X2007000200003\&ting=ptEtIng=es

Páramo, P. (2009). Pedagogía Urbana: elementos para su delimitación como campo de conocimiento. Revista Colombiana de Educación, 57 (2), 14-27.

Páramo, P. y Cuervo, M. (2009). La experiencia urbana en el espacio público de Bogotá, sigloxx. Universidad Pedagógica Nacional-Universidad Piloto de Colombia-Universidad Santo Tomás.

Páramo, P. y Mejía, M. (2004). Los parques urbanos como oportunidades para la interacción de los niños con los animales. Revista Latinoamericana de Psicología, 36 (1), 73-84.

Páramo, P. y Roa, E. (2015). La estructura conceptual de los miedos urbanos. Diversitas, 11 (1), 135-146.

Secretaría de Educación Distrital. (2015). Lineamientos Generales para la realización de Expediciones Pedagógicas para la Excelencia Académica y la Formación Integral. Alcaldía Mayor de Bogotá. 
Secretaría Distrital de Integración Social (sDIS). (2009). Lineamientos y Estándares Técnicos de Educación Inicial de la Secretaría Distrital de Integración Social. Alcaldia Mayor de Bogotá.

Tardif, M. (2004). Los saberes del docente y su desarrollo profesional. Narcea.

Tonucci, F. (2004). La ciudad de los niños. Fundación Germán Sánchez Ruipérez.
Tonucci, F. (2009). Ciudades a escala humana: la ciudad de los niños. Revista de Educación. Número extraordinario, 147-168.

Trilla, J. (2005). La idea de ciudad educadora y escuela. Educación y Ciudad, 7, 73-106.

Woodhead, M. y Oates, J. (2007). Primera infancia en perspectiva 1. Relaciones de apego. La calidad del cuidado en los primeros años. The Open University.

\section{Diálogo del conocimiento}

El presente artículo plantea aspectos muy importantes frente a las salidas pedagógicas en la educación inicial, los cuales deben tomar relevancia en los procesos y la programación de actividades que se proponen dia a dia en el aula escolar. Desde mi experiencia como maestra en educación infantil considero que las salidas pedagógicas representan una estrategia de aprendizaje fundamental para el desarrollo integral de los niños y las niñas, permiten la exploración, socialización, manejo de emociones, acercamiento a otros entornos y descubrimientos que hacen los niños y niñas a partir de la interacción con los diferentes elementos y objetos que encuentran en los ambientes que visitan como granjas, museos, parques, bibliotecas, ferias, entre otros. Por tanto, permiten y brindan la oportunidad de observar, manipular, reflexionar y constatar en la realidad aspectos que constantemente escuchan en el aula escolar.

Proponer salidas pedagógicas es una gran experiencia para los niños y las niñas. Desde el momento que la maestra menciona que se va a realizar una salida pedagógica, se generan expectativas e inquietudes en los niños; el solo hecho de pensar que irán a un lugar diferente al colegio y que van con sus amiguitos y profes mas no con sus padres, los niños comienzan a imaginar y a realizar inferencias de todo lo que va a pasar. Esto permite generar diversas actividades en torno a la salida pedagógica para responder preguntas como: profe, ¿a dónde vamos a ir?, ¿es lejos o cerca?, ¿Qué llevo en la maleta?, ¿Llevamos lonchera?, ¿Cuánto nos demoramos?, ¿Hace calor o frio?, ¿Nos vamos a quedar a dormir allá?, ¿Nos vamos en un bus grande? Asi van surgiendo diversas preguntas que permiten aprendizajes significativos y enriquecedores.

Como maestra de educación infantil, recuerdo esas lindas experiencias que he vivido con los niños al realizar las salidas pedagógicas, donde es evidente la huella significativa y el aprendizaje que deja en la vida de los niños. Es interesante que con artículos como este se continúe incitando y provocando a los maestros, maestras y directivos de las instituciones de educación inicial a proponer y programar con mayor constancia las salidas pedagógicas.

Sonia Maritza Prieto Buitrago

Maestra de Educación Infantil - Instituto Pedagógico Nacional 
